\title{
Nucleation and Growth of Precipitates in a V-Microalloyed Steel According to Physical Theory and Experimental Results
}

\author{
S. F. Medina ${ }^{a, *}$, P. Valles ${ }^{b}$, J. Calvo $^{c}$, and Jose M. Cabrera ${ }^{c}$ \\ ${ }^{a}$ National Centre for Metallurgical Research (CENIM-CSIC), Madrid, Spain \\ ${ }^{b}$ National Institute of Aerospace Technology (INTA), Torrejón de Ardoz, Madrid, Spain \\ ${ }^{c}$ Technical University of Catalonia (ETSEIB - UPC), Barcelona, Spain \\ *e-mail: smedina@cenim.csic.es \\ Received February 7, 2018; revised October 17, 2018; accepted August 9, 2019
}

\begin{abstract}
Using a theoretical model, the nucleus number and nucleation time were determined for a V-microalloyed steel. The calculated data has made it possible to plot the nucleus number vs. temperature, nucleation critical time vs. temperature, and precipitate critical radius vs. temperature. The nucleus number was calculated by integration of the nucleation rate expression. On the other hand, an experimental study was performed and the nucleation time vs. temperature was plotted (PTT diagram), thus allowing a comparison between the theoretical values and experimental results. It has been found that the growth of precipitates during precipitation obeys a quadratic growth equation and not a cubic coalescence equation. The experimentally determined growth rate coincides with the theoretically predicted growth rate. The experimental nucleation time is longer than the calculated time due to conceptual differences.
\end{abstract}

Keywords: V-microalloyed steel, growth of precipitates, nucleation time, precipitate

DOI: $10.1134 / \mathrm{S} 0031918 \mathrm{X} 2001010 \mathrm{X}$

\section{INTRODUCTION}

Microalloyed steels mainly contain small amounts of $\mathrm{Nb}$ and/or $\mathrm{V}$, and to a lesser extent $\mathrm{Ti}$, which being combined with $\mathrm{C}$ and $\mathrm{N}$ is prone to precipitate as carbides, nitrides, or carbonitrides. These precipitates modify the final microstructure obtained by hot deformation, favouring grain refinement and thus the mechanical properties of the steel. Ferrite grain refinement is achieved by prior hardening of the austenite due to the fact that the precipitates block static recrystallisation, raising the dislocation number density and thus favouring ferrite nucleation during cooling after deformation [1-3].

The recrystallisation/precipitation concurrent evolvement has been studied by many researchers, from the influence of the different variables that intervene in hot deformation to the construction of models that predict static recrystallisation, precipitation, or both [4-9]. Once nucleated, the precipitates can increase in size by growth and by coarsening or coalescence [10]. Both phenomena can take place simultaneously, although as will be seen in this work, that will depend on the temperature and the time.

In the literature it can be verified that there is no general agreement on the simultaneity of growth and coarsening of precipitates. By way of example, some authors indicate that the two phenomena do not take place at the same time [11-13] and others indicate the simultaneity of both $[14,15]$. As can be seen, if coars- ening occurs simultaneously with growth is a subject still under discussion.

Applying the classic theory of nucleation [16-18], the precipitation start curve, average nucleus radius, and precipitate size growth rate have been calculated in a V-microalloyed steel. Comparison of the calculated results and those obtained experimentally, along with the empirical model published by Medina et al. [19], helps to understand the precipitation phenomenon in its theoretical and experimental aspects, respectively.

\section{MATERIALS}

The steel was manufactured by continuous casting and hot rolling and its composition is shown in Table 1 , which includes an indication of the $\gamma \rightarrow \alpha$ transformation start temperature during cooling $\left(\mathrm{A}_{\mathrm{r} 3}\right)$, determined by dilatometry at a cooling rate of $0.2 \mathrm{~K} / \mathrm{s}$, which is the minimum temperature at which the different parameters related with austenite phase precipitation will be calculated.

\section{RESULTS AND DISCUSSION}

The nucleation rate is obtained from the classic theory of nucleation modified by Zeldovich, Kampamann, and Wagner as [18, 20]:

$$
\frac{d N}{d t}=N_{0} Z \beta^{\prime} \exp \left(-\frac{\Delta G}{k T}\right)\left(1-\exp \left(\frac{-t}{\tau}\right)\right)
$$


where, $N_{0}$ represents the number of available sites for heterogeneous nucleation, $Z$ is the Zeldovich nonequilibrium factor, $k$ is the Boltzman constant $\left(1.38065 \times 10^{-23} \mathrm{~J} \mathrm{~K}^{-1}\right), \beta^{\prime}$ is the rate at which the atoms are being added to the critical nucleus, or atomic impingement rate, $T$ is the absolute temperature, $\tau$ is the incubation period, and $\Delta G$ is the Gibbs energy of formation of a critical spherical nucleus of radius $R_{\mathrm{c}}$.

The integration of Eq. (1) between time zero and $\tau$ would give the number of nuclei that have formed during the incubation period, before they start growing as precipitation progresses. The integrated equation should be:

$$
\begin{aligned}
N= & N_{0} Z \beta^{\prime} \exp \left(-\frac{\Delta G}{k T}\right)\left[t+\tau \exp \left(-\frac{t}{\tau}\right)\right]_{0}^{\tau} \\
& =N_{0} Z \beta^{\prime} \exp \left(-\frac{\Delta G}{k T}\right)\left(\frac{\tau}{2.718}\right),
\end{aligned}
$$

where, $N$ represents the number of precipitates per unit volume.

Gibbs energy $(\Delta G)$ for the formation of a spherical nucleus of carbonitride from the elements in solution $(\mathrm{V})$ is classically expressed as the sum of chemical free energy, interfacial free energy, and dislocation core energy, resulting in the following expression $[10,16,21]$ :

$$
\Delta G=\frac{16 \pi \gamma^{3}}{3 \Delta G_{V}^{2}}+0.8 \mu b^{2} \frac{\gamma}{\Delta G_{V}} .
$$

where, $\Delta G$ is given in $\mathrm{J}, \gamma$ is the surface energy of the precipitate $\left(0.5 \mathrm{~J} \mathrm{~m}^{-2}\right)$, and $\Delta G_{V}$ is the driving force for nucleation $\left(\mathrm{J} \mathrm{m}^{-3}\right)$ of precipitates, $b$ is the Burgers vector abs. value of austenite, and $\mu$ is the shear modulus. For austenite, $b=2.59 \times 10^{-10} \mathrm{~m}$ and $\mu=4.5 \times 10^{4} \mathrm{MPa}$ [10]. The second term of expression (3) is negative due to the fact that $\Delta G_{V}$ is negative, as can be seen in Eq. (4).

The equilibrium between the austenite matrix and the carbonitride $\mathrm{VC}_{y} \mathrm{~N}_{1-y}$ is described by the mass action law [14]:

$$
=-\frac{R_{\mathrm{g}} T}{V_{\mathrm{m}}}\left[\ln \left(\frac{X_{\mathrm{V}}^{\mathrm{ss}}}{X_{\mathrm{V}}^{\mathrm{e}}}\right)+y \ln \left(\frac{X_{\mathrm{C}}^{\mathrm{ss}}}{X_{\mathrm{C}}^{\mathrm{e}}}\right)+(1-y) \ln \left(\frac{X_{\mathrm{N}}^{\mathrm{ss}}}{X_{\mathrm{N}}^{\mathrm{e}}}\right)\right],
$$

where, $X_{i}^{\text {ss }}$ are the molar fractions in solid solution of $\mathrm{V}, \mathrm{C}$, and $\mathrm{N}$, respectively, and $X_{i}^{\mathrm{e}}$ are the equilibrium fractions at the deformation temperature, $V_{\mathrm{m}}$ is the molar volume of precipitate species, $R_{\mathrm{g}}$ is the universal gas constant $\left(8.3145 \mathrm{~J} \mathrm{~mol}^{-1} \mathrm{~K}^{-1}\right)$, and $T$ is the deformation absolute temperature.

The carbonitride is considered as an ideal mix of $\mathrm{VC}$ and $\mathrm{VN}$ and the values of parameters $y ; X_{\mathrm{V}}^{\mathrm{e}} ; X_{\mathrm{C}}^{\mathrm{e}} ; X_{\mathrm{N}}^{\mathrm{e}}$ are determined using FactSage $[22,23]$. The value of " $y$ " in equation (4) is the precipitated $\mathrm{VC} / \mathrm{VCN}$ ratio, and " $1-y$ " is the $\mathrm{VN} / \mathrm{VCN}$ ratio, being $\mathrm{VCN}=\mathrm{VC}+\mathrm{VN}$.
Table 1. Chemical composition (wt \%) and transformation critical temperature $\left(\mathrm{A}_{\mathrm{r} 3}, 0.2 \mathrm{~K} / \mathrm{s}\right)$

\begin{tabular}{c|c|c|c|c|c}
\hline $\mathrm{C}$ & $\mathrm{Si}$ & $\mathrm{Mn}$ & $\mathrm{V}$ & $\mathrm{N}$ & $\mathrm{Al}$ \\
\hline 0.10 & 0.30 & 1.46 & 0.139 & 0.0100 & 0.026 \\
$\mathrm{~A}_{\mathrm{r} 3}=1050 \mathrm{~K}\left(777^{\circ} \mathrm{C}\right)$
\end{tabular}

On the other hand, $N_{0}=0.5 \Delta \rho^{1.5}$ is the number of nodes in the dislocation network, $\Delta \rho=(\Delta \sigma / 0.2 \mu b)^{2}$ is the variation in the number density of dislocations associated with the recrystallisation front movement in the deformed zone at the start of precipitation [10], and $\Delta \sigma$ is the difference between the flow stress and yield stress at the deformation temperature.

The atomic impingement rate is given as [16]:

$$
\beta^{\prime}=\frac{4 \pi R_{\mathrm{c}}^{2} D_{\mathrm{V}} C_{\mathrm{V}}}{a^{4}},
$$

where, $\beta^{\prime}$ is given in $\mathrm{s}^{-1}, R_{c}$ is the critical radius for nucleation, $D_{\mathrm{V}}$ is the bulk diffusivity of solute atoms (V) in the austenite, $a$ is the lattice parameter of the precipitate, and $C_{\mathrm{V}}$ is the initial concentration of vanadium in mol fraction. Here, the bulk diffusion coefficient $\left(D_{\mathrm{V}}\right)$ is replaced by an effective diffusion coefficient, $D_{\text {eff }}$, expressed as a weighted mean of the bulk diffusion $\left(D_{\mathrm{V}}\right)$ and pipe diffusion $\left(D_{\mathrm{p}}\right)$ coefficients, and used in the description of the precipitate evolution [16]:

$$
D_{\text {eff }}=D_{p} \pi R_{\text {core }}^{2} \rho+D_{\mathrm{V}}\left(1-\pi R_{\text {core }}^{2} \rho\right),
$$

where, $R_{\text {core }}$ is the radius of the dislocation core, taken to be equal to the burgers vector modulus, $b$.

The effective diffusivity term is also used instead of the bulk diffusivity term to determine the growth rate, because growth is significantly affected by pipe diffusion through dislocation cores when precipitates nucleate on dislocations [15].

The Zeldovich factor $\mathrm{Z}$ takes into account that the nucleus is destabilised by thermal excitation compared to the inactivated state and is given as [24]:

$$
Z=\frac{V_{\mathrm{at}}^{\mathrm{p}}}{2 \pi R_{\mathrm{c}}^{2}} \sqrt{\frac{\gamma}{k T}},
$$

where, $V_{\text {at }}^{\mathrm{p}}$ is the molecular volume of precipitate.

The flow stress increment $(\Delta \sigma)$ has been calculated using the model reported by Medina and Hernández, which facilitates the calculation of flow stress [25]. The dislocation density has been calculated at the nose temperature of the $P_{\mathrm{s}}$ curves corresponding to a strain of 0.35 . When the austenite is not deformed the dislocation density is approximately $10^{12} \mathrm{~m}^{-2}$ [26] and the dislocation density corresponding to the curve nose will be given by $10^{12}+\Delta \rho$.

On the other hand, the term $1-\exp (-t / \tau)$ in equation (1) indicates the progress of the nucleation rate, 


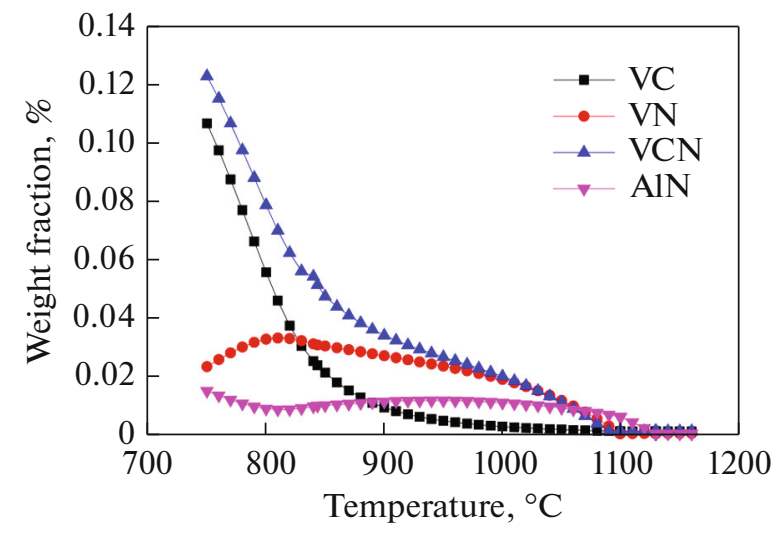

Fig. 1. Equilibrium compounds of steel used predicted by FactSage.

but most authors do not take this into account, as the incubation period is very short.

In Eq. (1) the incubation time $(\tau)$ is given as follows [27]:

$$
\tau=\frac{1}{2 \beta^{\prime} Z^{2}} \text {. }
$$

The critical radius for nucleation is determined from the driving force and is given as [14]:

$$
R_{\mathrm{c}}=-\frac{2 \gamma}{\Delta G_{v}} \text {. }
$$

It is obvious that the nuclei that can grow have to be bigger than the critical nucleus and in accordance with Dutta et al. [10] and Perez et al. [28] this value will be multiplied by 1.05 . It must be noted that the factor 1.05 has little consequence on the overall precipitation kinetics.

During precipitation, and once the nuclei have formed with the critical size given by Eq. (9) multiplied by factor 1.05 , precipitate growth will take place during precipitation and will start once the nucleation time given by equation (8) has passed. From the mass balance at the precipitate/matrix interface the classical Zener equation for the growth rate is fulfilled [14]:

$$
\frac{d R}{d t}=\frac{D_{\mathrm{eff}}}{R} \frac{X_{\mathrm{V}}^{\mathrm{ss}}-X_{\mathrm{V}}^{\mathrm{i}}}{\frac{V_{\mathrm{Fe}}}{V_{\mathrm{p}}}-X_{\mathrm{V}}^{\mathrm{i}}},
$$

where, $V_{\mathrm{Fe}}$ is the molar volume of austenite, $V_{\mathrm{p}}$ is the molar volume of precipitate, and $X_{\mathrm{V}}^{\mathrm{i}}$ is the content of vanadium at the interface.

Carbon and nitrogen, being interstitial elements, are very fast-diffusing species compared to vanadium. As a result, the growth of a precipitate is limited by the diffusion of vanadium from the matrix to the precipitate. During isothermal precipitation treatment the content of vanadium in solid solution $X_{\mathrm{V}}^{\mathrm{ss}}$ decreases, and consequently the growth rate decreases too. In the meantime, the content of vanadium at the interface $X_{\mathrm{V}}^{\mathrm{i}}$ evolves.

The integration of equation (10) will give the radius of the precipitates as a function of time:

$$
R^{2}=R_{0}^{2}+2 D_{\text {eff }} \frac{X_{\mathrm{V}}^{\mathrm{SS}}-X_{\mathrm{V}}^{\mathrm{i}}}{\frac{V_{\mathrm{Fe}}}{V_{\mathrm{p}}}-X_{\mathrm{V}}^{\mathrm{i}}} t,
$$

where, $R$ is the precipitate radius after a certain time $t$ and $R_{0}$ is the average critical radius of the precipitates that have nucleated during the incubation period, coinciding with $1.05 R_{0}$, which is stable, it does not dissolve, and it can start to grow.

Coarsening is the process whereby the smallest precipitates dissolve to the benefit of the larger ones, leading to a coarsening of the size distribution. This phenomenon is particularly important when the system reaches the equilibrium precipitate fraction. It is to be noted that coarsening can occur at every stage of the precipitation process [14]. The last process is known as Ostwald ripening according to Lifshitz and Slyozov's expression $[29,30]$ :

$$
R^{3}=R_{0}^{3}+\frac{8 D_{\mathrm{v}} \gamma V_{p}^{2} X_{\mathrm{V}}^{\mathrm{i}}}{9 R_{\mathrm{g}} T} t,
$$

where, $R_{\mathrm{g}}$ is the gas constant and the other parameters have already been explained.

The most striking aspect of Eq. (12) is that the mean radius cubed varies with time, as opposed to the radius squared in growth calculations. Coarsening is thus a much slower process than precipitate growth, as is reasonable since the growth of one particle only occurs at the expense of dissolving other particles. The theory also predicts that the system of particles will take on a time-independent size distribution, with a limiting particle size of, $R_{\max }=1.5 \bar{R}$.

The FactSage software tool for the calculation of phase equilibria and thermodynamic properties makes it possible to predict the formation of simple precipitates (nitrides and carbides) and more complex precipitates (carbonitrides) and the results can be expressed as a weight fraction versus temperature. Figure 1 shows the fraction of $\mathrm{AIN}, \mathrm{CV}, \mathrm{VN}$, and total $\mathrm{CVN}$ versus the temperature. These results are thermodynamic predictions and should not be interpreted as real results obtained at tested deformation temperatures. The precipitated fraction of AlN is above VCN until $1323 \mathrm{~K}$ $\left(1050^{\circ} \mathrm{C}\right)$ and at lower temperatures the volume of AlN starts to be much less important. Furthermore, AlN starts to precipitate before CVN, approximately at $1403 \mathrm{~K}\left(1130^{\circ} \mathrm{C}\right)$. The precipitated volume of AIN particles is small and coincides with the results found by other authors for relatively low contents of $\mathrm{Al}$ as in the present case which is $0.026 \mathrm{wt} \%[30,31]$. 


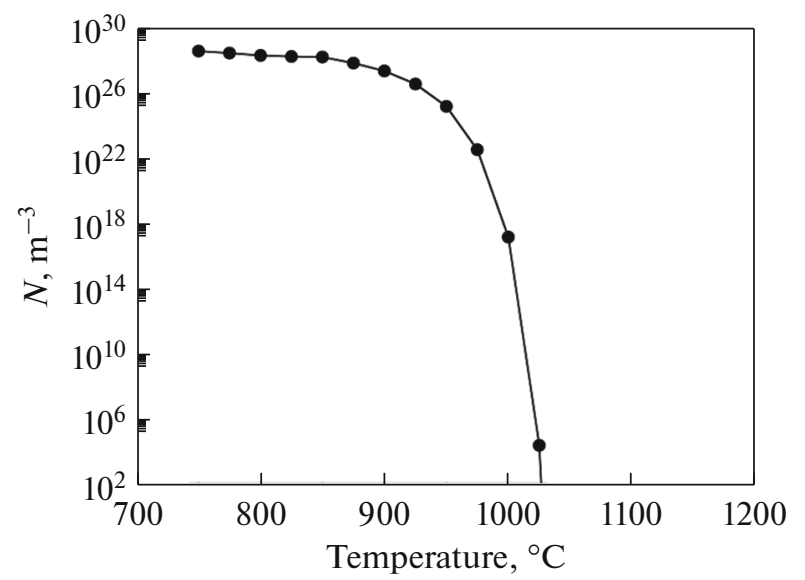

Fig. 2. Precipitate density number vs temperature until incubation period $(\tau)$.

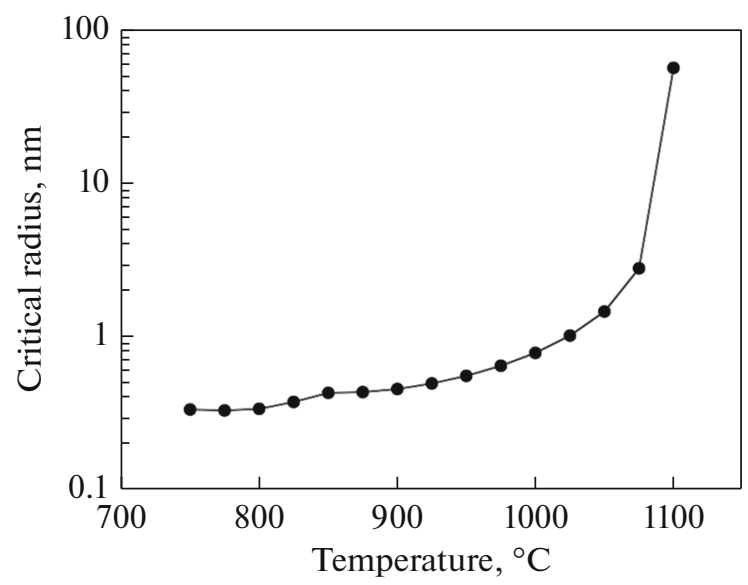

Fig. 4. Critical radius size vs temperature.

Figure 2 shows the number of precipitates $(N)$ versus temperature in accordance with equation (2), at incubation times given by Eq. (8). The value of $N$ increases constantly as the temperature decreases, but only until the incubation time is completed, because a possible dissolution of those particles that have not reached the critical radius and possible coarsening of stable particles, which would cause the value of $N$ to decrease, take place after the end of the incubation period. Consideration of the dislocation core energy and the effective diffusion coefficient $\left(D_{\text {eff }}\right)$ instead of only the bulk diffusion coefficient $\left(D_{\mathrm{V}}\right)$ has led to a considerable increase in the value of $N[20,24]$.

The values calculated for the incubation period $(\tau)$ are shown as a function of the temperature (Fig. 3). As the effective energy was taken into account in Eq. (4) instead of only the energy for bulk diffusion, the values of $\tau$ were lower. For their part, the minimum nucleation times achieved correspond to temperatures close to $1273 \mathrm{~K}\left(1000^{\circ} \mathrm{C}\right)$. The critical radius $\left(R_{\mathrm{c}}\right)$ for

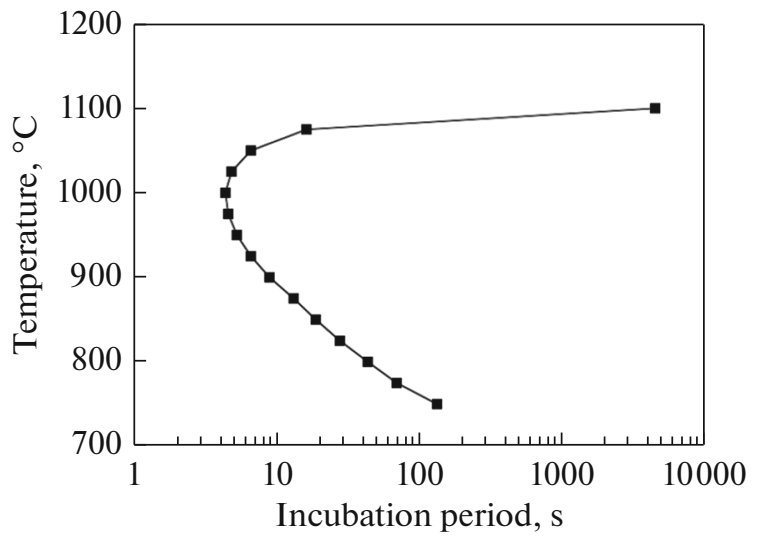

Fig. 3. Incubation period vs temperature (curve $P_{\mathrm{s}}$ ).

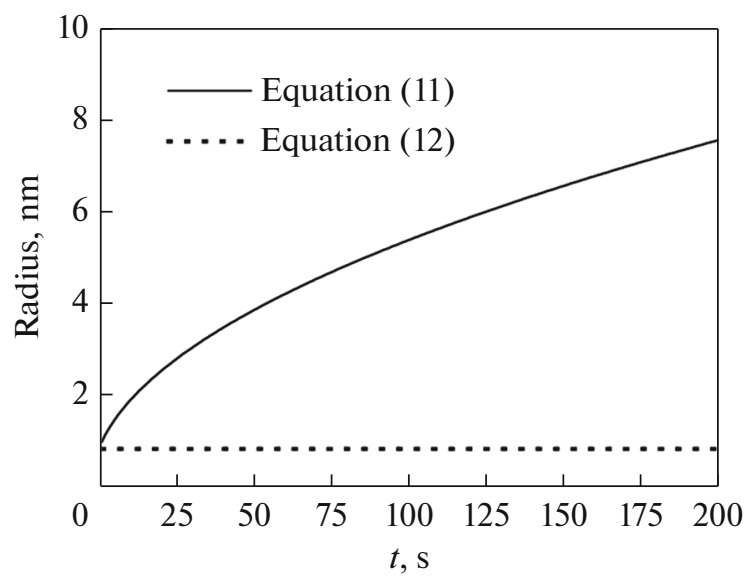

Fig. 5. Precipitate size (diameter) vs time $(t>\tau)$.

nucleation was calculated from equation (9) as a function of the temperature (Fig. 4).

Finally, Eqs. (11) and (12) have been applied to the precipitate growth and coarsening, respectively, selecting a temperature of $1273 \mathrm{~K}\left(1000^{\circ} \mathrm{C}\right)$ which corresponds to that of the curve nose (Fig. 3). The time $(t)$ starts to be counted after the nucleation period, which was $4.5 \mathrm{~s}$ at this temperature. The value of $R_{0}$ was $0.78 \mathrm{~nm}$ (Fig. 4), multiplied by 1.05 . The results are shown in Fig. 5, where it can be seen that the application of the quadratic growth model (Eq. (11)) allows prediction of the increase in the precipitate size.

However, the cubic coarsening model (Eq. (12)) does not predict the increase in precipitate size, being constant versus time. This is due mainly to the fact that the value of the coefficient $D_{\text {eff }}$ varies strongly with the temperature and its calculated value of $5.22 \times 10^{-16} \mathrm{~m}^{2} \mathrm{~s}^{-1}$, corresponding to $1000^{\circ} \mathrm{C}$, is relatively low.

The final moment of precipitation is given by the precipitated fraction (Fig. 1), and the final precipitate radius is determined by simple calculation taking into 


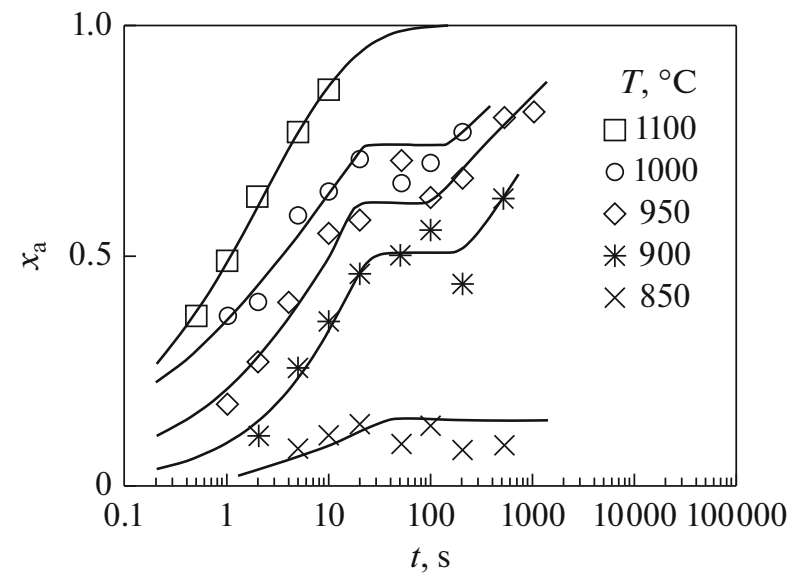

Fig. 6. Recrystallised fraction against time. Strain $=0.35$, strain rate $=3.63 \mathrm{~s}^{-1}$; Reh. Temp. $=1200^{\circ} \mathrm{C}$.

account the precipitate density $\left(6000 \mathrm{~kg} / \mathrm{m}^{3}\right)$ and austenite density $\left(8150 \mathrm{~kg} / \mathrm{m}^{3}\right)$. This calculation indicates that a good part of the precipitates do not grow, especially when the temperature drops, and are newly dissolved.

If this were not so, and all the nuclei that form were to grow, the amount of the precipitated fraction would be much greater than that corresponding to the weight percentage shown in Fig. 1. According to Deschamps and Brechet [32], there is a progressive transition from the nucleation/growth regime to the growth/coarsening regime. A simple criterion for this transition is when the decrease in precipitate density by coarsening is larger than the increase in precipitate density by nucleation, i.e.

$$
-\left.\frac{d N}{d t}\right|_{\text {growth }+ \text { coars }}>\left.\frac{d N}{d t}\right|_{\text {nucleation }} .
$$

The specimens for torsion tests had a gauge length of $50 \mathrm{~mm}$ and a diameter of $6 \mathrm{~mm}$. The austenitisation temperature was $1473 \mathrm{~K}\left(1200^{\circ} \mathrm{C}\right)$ for 10 minutes. The statically recrystallised fraction was determined for several temperatures between $1373 \mathrm{~K}\left(1100^{\circ} \mathrm{C}\right)$ and $1073 \mathrm{~K}\left(800^{\circ} \mathrm{C}\right)$, applying the double deformation technique, and, in particular, the back extrapolation method [5].

Comparison of Figs. 2 and 3 shows that the number of nuclei formed as a function of temperature is small at the curve nose temperature $\left(T_{\mathrm{N}}=1273 \mathrm{~K} ; 1000^{\circ} \mathrm{C}\right)$. In other words, the theoretically calculated nucleation time is a little high or the formation of nuclei should be slightly higher at the $T_{\mathrm{N}}$ temperature. The calculations have been performed using the parameters in Table 2 .

The recrystallised fraction $\left(X_{\mathrm{a}}\right)$ was plotted against time, obtaining curves such as those included in Fig. 6, which correspond to a strain of 0.35 . Static recrystallisation (SRX) occurs after hot deformation, while the stress or strain is below the critical value for the onset of dynamic recristallisation (DRX) [33].

The curves corresponding to temperatures equal to or below $1273 \mathrm{~K}\left(1000^{\circ} \mathrm{C}\right)$ present a plateau, or the inhibition of recrystallisation, due to strain-induced precipitation of $\mathrm{V}(\mathrm{C}, \mathrm{N})$ particles. The curve that does not present a plateau has the typical sigmoidal shape of Avrami's law.

The length of the plateau is not unlimited, and after a short inhibition time recrystallisation once again progresses until reaching completion. The recommencement of recrystallisation is due fundamentally to the reduction of pinning forces by the growth of particles during precipitation, versus the driving forces for recrystallisation. The start and end of the plateau are approximately identified with the start and end of precipitation. It is conventionally accepted to estimate a $5 \%$ precipitated fraction for the start and a $95 \%$ fraction for the end [34].

Table 2. Parameters used for calculations

\begin{tabular}{l|l|c|c}
\hline \multicolumn{1}{c|}{ Parameter } & \multicolumn{1}{c|}{ Symbol } & Value & Reference \\
\hline Burgers vector abs. value & $b, \mathrm{~m}$ & $2.59 \times 10^{-10}$ & {$[10]$} \\
Shear modulus & $\mu, \mathrm{MPa}$ & $4.5 \times 10^{4}$ & {$[10]$} \\
Interfacial energy & $\gamma, \mathrm{J} \mathrm{m}^{-2}$ & 0.5 & {$[14,16]$} \\
Lattice parameter $(\mathrm{VCN})$ & $a, \mathrm{~nm}$ & 0.4118 & {$[15]$} \\
Bulk diffusion of V & $D_{\mathrm{V}}, \mathrm{m}^{2} \mathrm{~s}^{-1}$ & $0.28 \times 10^{-4} \mathrm{exp}(-264000 / R T)$ & {$[29]$} \\
Pipe diffusion & $D_{\mathrm{p}}, \mathrm{m}^{2} \mathrm{~s}^{-1}$ & $0.25 \times 10^{-4} \mathrm{exp}(-210000 / R T)$ & {$[15]$} \\
Molar volume of VCN & $V_{\mathrm{P}}, \mathrm{m}^{3} \mathrm{~mol}^{-1}$ & $10.65 \times 10^{-6}$ & {$[14]$} \\
Molar volume of austenite & $V_{\mathrm{Fe}}, \mathrm{m}^{3} \mathrm{~mol}^{-1}$ & $7.11 \times 10^{-6}$ & {$[14]$} \\
Dislocation core radius & $R_{\mathrm{core}}, \mathrm{m}^{-6}$ & $5.00 \times 10^{-10}$ & {$[16]$} \\
Molecular volume of precipitate & $V_{\mathrm{at}}^{\mathrm{p}}, \mathrm{m}^{3}$ & $1.7468 \times 10^{-29}$ & {$[26]$} \\
\hline
\end{tabular}


It is known that once the plateau has ended, and recrystallisation is again progressing, the pinning forces exerted by the precipitates are now lower than the driving forces for recrystallisation. The "recrystallised fraction versus time" curves were used to plot the precipitation-time-temperature (PTT) diagram of the steel used [34]. The points defining the start and the end of the plateau were taken to plot the curves for the start $\left(P_{\mathrm{s}}\right)$ and the end $\left(P_{\mathrm{f}}\right)$ of precipitation, respectively. In this way, the PTT diagram was obtained for a strain $(\varepsilon)$ of 0.35 and strain rate $(\dot{\varepsilon})$ of $3.63 \mathrm{~s}^{-1}$, as shown in Fig. 7.

The most important magnitudes that can be deduced from a PTT diagram, and which are also indispensable for the perfect configuration of the diagram, are the aforementioned minimum incubation time $\left(t_{\mathrm{N}}\right)$, curve nose temperature $\left(T_{\mathrm{N}}\right)$ and the precipitation end time $\left(t_{\mathrm{N}}^{\prime}\right)$ at the nose temperature (Table 3 ).

Once the $P_{\mathrm{f}}$ curve has been reached, recrystallisation starts to progress again due to fact that the pinning forces exerted by the precipitates are lower than the driving forces for recrystallisation.

The PTT diagrams, and especially the $P_{\mathrm{s}}$ and $P_{\mathrm{f}}$ curves, define a time interval, whatever the temperature, during which the precipitation state (size and precipitated volume) is changing. For times after $P_{f}$, the precipitated fraction does not vary but a coarsening of the precipitates occurs due to the effect of Ostwald ripening.

The start of the plateau in the recrystallised fraction is identified with the start of precipitation and its duration is related with two phenomena that take place simultaneously, namely increases in the precipitated volume and in the size of the precipitates.

Several studies carried out with the assistance of transmission microscopy (TEM) have shown the speed at which the coarsening of precipitates occurs and indicated how the size distribution changes from the start of the plateau to its end [9]. Figure 8 shows the TEM resolution on carbon extraction replicas of vanadium nitride particles corresponding to the steel used, obtained on specimens strained and quenched in the conditions indicated at the foot of the figure.

On the other hand, the particle size distribution and determination of the average particle size have been obtained by measuring an average number of 200 particles on the specimen. Figure 9 shows a lognormal distribution which corresponds to times of 20 and $100 \mathrm{~s}$, close to the start and end of the plateau, respectively, with a weighted mean size $(D)$ of 6.4 and $9.5 \mathrm{~nm}$ and two maximum frequencies of $6 \mathrm{~nm}$ for both (Table 4). On the other hand, the evolution of the precipitate size can be modelled following a law of the type shown in Eq. (11), such as:

$$
R^{2}=R_{0}^{2}+M t
$$

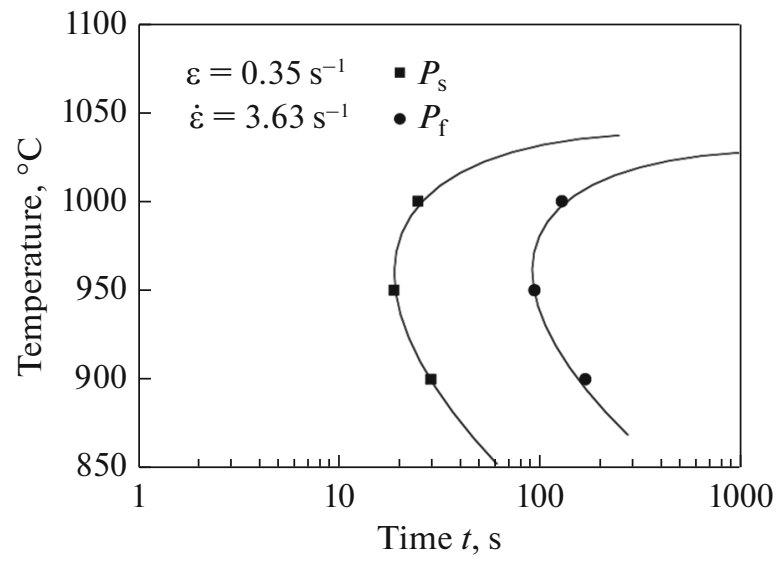

Fig. 7. Experimental PTT diagram for steel used.

where, $R$ is the precipitate radius $(R=\bar{D} / 2)$ given in $\mathrm{m}$, $R_{0}$ is the nucleus radius, and $M$ is a constant that depends on the temperature.

If we accept that the precipitates grow once they are nucleated, then moment zero of growth coincides with the $P_{\mathrm{s}}$ curve and $\Delta t$ will be the sample holding time after $P_{\mathrm{s}}$.

Figure 10 shows a representation of the average precipitate growth at a temperature of $1223 \mathrm{~K}\left(950^{\circ} \mathrm{C}\right)$, close to the experimental $T_{\mathrm{N}}$ temperature $(1228 \mathrm{~K}$; $955^{\circ} \mathrm{C}$ ). The holding times after deformation were 20 and $100 \mathrm{~s}$, minus the $P_{\mathrm{s}}$ time corresponding to $1223 \mathrm{~K}$ $\left(950^{\circ} \mathrm{C}\right)$, coinciding with $t_{\mathrm{N}}$ which was $19 \mathrm{~s}$. In accordance with equation (14) the following equation was obtained:

$$
R^{2}=1 \times 10^{-17}+0.015 \times 10^{-17} t .
$$

From equation (15) it is possible to deduce the nucleus radius $\left(R_{0}\right)$, which was $3.1 \mathrm{~nm}$. On the other hand, the mean size of strain-induced AlN precipitates (Fig. 1) is almost one order of magnitude bigger than the size of VCN particles. The higher solubility temperatures of AlN along with the larger diffusion coefficient in austenite of $\mathrm{Al}$ compared to $\mathrm{V}$ are the main reasons for the coarser size of AlN particles. The pinning forces exerted by coarse AlN precipitates are weak and accordingly the static recrystallisation of austenite is not inhibited by these particles [35]. The calculated minimum incubation time $\left(t_{\mathrm{N}}\right)$ was $4.5 \mathrm{~s}$ and the temperature $\left(T_{\mathrm{N}}\right)$ was $1273 \mathrm{~K}\left(1000^{\circ} \mathrm{C}\right)$ (Fig. 3). The experimental values were $19 \mathrm{~s}$ and $1228 \mathrm{~K}$

Table 3. Experimental minimum incubation time $\left(t_{\mathrm{N}}\right)$, curve nose temperature $\left(T_{\mathrm{N}}\right)$ and precipitation duration time

\begin{tabular}{c|c|c}
\hline$t_{\mathrm{N}}, \mathrm{s}$ & $t_{\mathrm{N}}^{\prime}, \mathrm{s}$ & $T_{\mathrm{N}}, \mathrm{K}\left({ }^{\circ} \mathrm{C}\right)$ \\
\hline 19 & 95 & $1228(955)$ \\
\hline
\end{tabular}


(a)

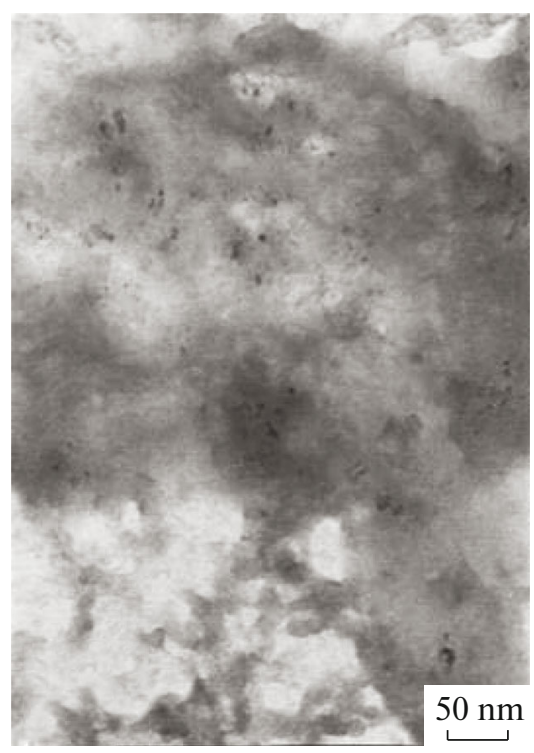

(b)

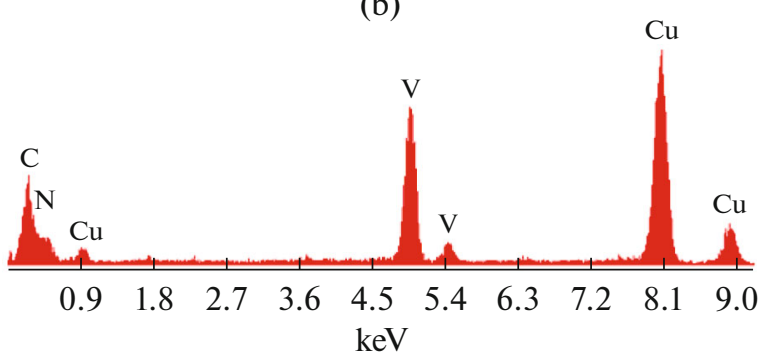

(c)

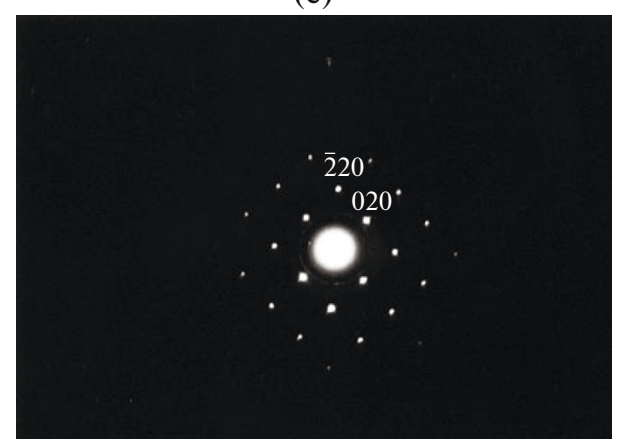

Fig. 8. TEM images. (a) Image showing precipitates for specimen tested at deformation temperature of $950^{\circ} \mathrm{C}$ and holding time $=20 \mathrm{~s}$. (b) EDAX spectrum of precipitate.

(c) Electron diffraction image.

$\left(955^{\circ} \mathrm{C}\right)$, respectively. Comparison of the calculated and experimental values shows an approximation of the temperature and greater discrepancy in the incubation time. Nevertheless, this difference may be explained bearing in mind the recent work of Medina et al. [36] which shows that the influence of the strain on the incubation time depends on the microalloying content, whereby the influence exerted is greater, the lower the microalloying content. In other words, when the driving force for nucleation of precipitates $\left(\Delta G_{\mathrm{v}}\right)$ is

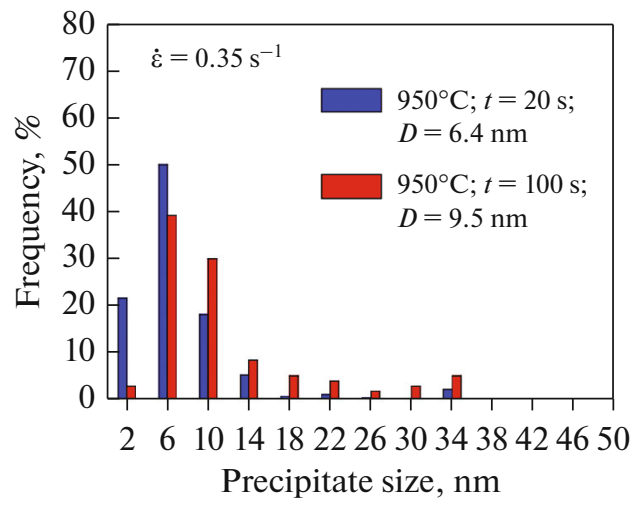

Fig. 9. Size distribution of precipitates for specimen tested at reheating temperature of $1200^{\circ} \mathrm{C}$ for $10 \mathrm{~min}$, deformation temperature of $950^{\circ} \mathrm{C}$ and recrystallisation times 20 and $100 \mathrm{~s}$.

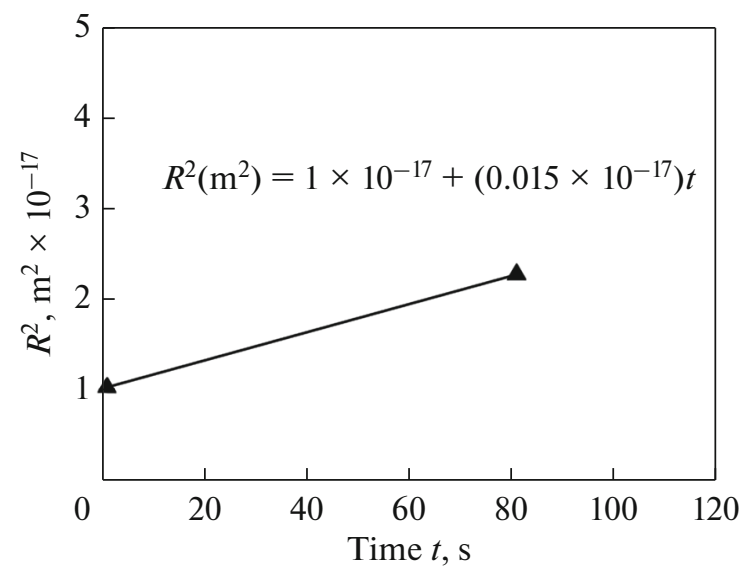

Fig. 10. Experimental growing of precipitate.

high, or low in absolute terms, the strain as a promoter of the increase in the dislocation density exerts an important influence on the incubation time. In contrast, when the driving force is low, or high in absolute terms, the strain loses its influence on the nucleation time and homogenous nucleation is preponderant over heterogeneous nucleation.

The value for $\Delta G_{\mathrm{v}}$ calculated using Eq. (4) at the nose temperature $\left(T_{\mathrm{N}}=1228 \mathrm{~K} ; 955^{\circ} \mathrm{C}\right)$ was $-1.554 \times$ $10^{9} \mathrm{~J}^{-3}$. According to Medina et al. $[19,36]$ the incubation time corresponding to $5 \%$ of the precipitated volume $\mathrm{t}_{0.05}$, which in practical terms can be taken as the nucleation period for precipitation, depends on the strain as follows:

$$
t_{0.05} \infty \varepsilon^{-\alpha}
$$

where, $\alpha$ has the following expression:

$$
\alpha=1.96\left[1-\exp \left(-3.994 \times 10^{-2}\left(\frac{1}{w}\right)^{0.813}\right)\right],
$$


with $w$ the microalloying element content (wt \%).

The exponent $\alpha$ can be calculated according to expression (16), obtaining the value of 0.34 . This value indicates that the applied strain has very little influence on the incubation time as it is very low compared to the maximum value $(\alpha=2)$ found by Medina et al. [19] in steels with a low microalloying content, which means that the nucleation of the precipitates has been possibly more homogenous than heterogeneous [36].

On the other hand, the calculated and experimental nucleation times do not have the same meaning. The nucleation time calculated means the time necessary for the formation of a number $N$ of nuclei, of size $R_{0}$, whose growth continues when the precipitate radius reaches a size of $1.05 R_{0}$. The experimental nucleation time refers to the time necessary for the pinning forces to exceed the driving forces for recrystallisation temporarily inhibiting the progress of recrystallisation. It is logical to think that the experimental incubation time will always be longer than the calculated inhibition time.

The average experimentally measured nucleus radius was $3.1 \mathrm{~nm}$ at $1223 \mathrm{~K}\left(950^{\circ} \mathrm{C}\right)$, which is greater than the nucleus radius calculated at the theoretical curve nose, which was approximately $0.8 \mathrm{~nm}$. Though of the same order, it is again necessary to highlight the conceptual difference between the theoretical and experimental $P_{\mathrm{s}}$ curves, respectively.

Experimental Eq. (15) shows that the growth in the precipitate size from the start of precipitation ( $P_{\mathrm{s}}$ curve) to the end of precipitation $\left(P_{\mathrm{f}}\right.$ curve $)$ can be explained by means of a growth equation with a slope equal to $0.015 \times 10^{-17}$. On the other hand, from the holding time of $20 \mathrm{~s}$ to the holding time of $100 \mathrm{~s}$ the measured precipitate radius (Table 4 ) has increased from 3.2 to $4.7 \mathrm{~nm}$, an increase of $1.5 \mathrm{~nm}$. If the times of $20 \mathrm{~s}$ and $100 \mathrm{~s}$ are inserted in Fig. 5, subtracting from both the calculated incubation time $(4.5 \mathrm{~s})$, it is seen that the nucleus radius increases from 2.5 to $5.3 \mathrm{~nm}$, which is very similar to the experimental values. It is therefore deduced from both the experimental and theoretical points of view that the increase in precipitate size between $P_{\mathrm{s}}$ and $P_{\mathrm{f}}$ can be predicted by the growth Eq. (11), as the coarsening given by Eq. (12) is not taking place.

Finally, other authors have measured the sizes of VCN precipitates in a forged and cooled V-steel with no time for growth, finding that $95 \%$ had sizes of less than $5 \mathrm{~nm}$, coinciding with the sizes determined in this work at the start of nucleation [37].

In the present work, we do not reach the conclusion that the growth occur or not simultaneously with the coarsening, only is affirmed that for the test temperature $950^{\circ} \mathrm{C}$, the coarsening Eq. (12) is constant in the estimated calculation time (200 s). However, Eq. (11) for growth gives a noticeable increase in radius of the particle. On the other hand for very long times and especially for higher temperatures Eq. (12) would give an increasing of precipitate. Some authors
Table 4. Precipitate size (diameter) for different holding times after deformation at $950^{\circ} \mathrm{C}$

\begin{tabular}{c|c}
\hline Holding time, $\mathrm{s}$ & Diameter, $\mathrm{nm}$ \\
\hline 20 & 6.4 \\
100 & 9.5 \\
\hline
\end{tabular}

have experimentally shown that at high austenization temperatures, but lower than the solubility temperature, as is the case of steels with $\mathrm{Ti}$ as a micro-alloying element, a marked coarsening of the precipitates occurs [38].

\section{CONCLUSIONS}

The theoretically determined $P_{\mathrm{s}}$ precipitation start curve differs from the experimental $P_{\mathrm{s}}$ curve. The theoretical and experimental minimum times (curve nose) were 4.5 and $19 \mathrm{~s}$, respectively. This difference has been explained taking into account the conceptual difference between the experimental and theoretical $P_{\mathrm{s}}$ curves, which explains why the experimental nucleation times are always higher than the calculated times.

The calculated average nucleus radius was approximately $0.8 \mathrm{~nm}$ at the theoretical $P_{\mathrm{s}}$ curve nose temperature $\left(1273 \mathrm{~K} ; 1000^{\circ} \mathrm{C}\right)$ and the experimental average radius was $3.1 \mathrm{~nm}$ at the temperature of $1223 \mathrm{~K}$ $\left(950^{\circ} \mathrm{C}\right)$, close to the experimental curve nose temperature $\left(1228 \mathrm{~K} ; 955^{\circ} \mathrm{C}\right)$. Nevertheless, both sizes are practically the same when similar holding times are considered.

The precipitate size growth rate between the experimentally determined $P_{\mathrm{s}}$ and $P_{\mathrm{f}}$ virtually coincides with the calculated growth rate. Both are expressed by a quadratic growth equation.

\section{ACKNOWLEDGMENT}

The authors gratefully acknowledge the financial support of Spanish Ministry of Economy and Competitiveness through the project ref. MAT2011-29039-C02-02.

\section{REFERENCES}

1. K. Xu, B. G. Thomas, and R. O'Malley, "Equilibrium model of precipitation in microalloyed steels," Metall. Mater. Trans. A 42, 524-539 (2011).

2. C. M. Sellars, "Modelling microstructural development during hot rolling," Mater. Sci. Technol. 6, 10721081 (1990).

3. W. J. Liu, "A new theory and kinetic modelling of strain-induced precipitation of $\mathrm{Nb}(\mathrm{CN})$ in microalloyed austenite," Metall. Mater. Trans. A 26, 16411657 (1995).

4. M. Gómez, S. F. Medina, A. Quispe, and P. Valles, "Static recrystallization and induced precipitation in a low $\mathrm{Nb}$ microalloyed steel," ISIJ Int. 42, 423-431 (2002). 
5. H. L. Andrade, M. G. Akben, and J. J. Jonas, "Effect of molybdenum, niobium, and vanadium on static recovery and recrystallization and on solute strengthening in microalloyed steels," Metall. Trans. A 14, 19671977(1983).

6. O. Kwon, "A technology for the prediction and control of microstructural changes and mechanical properties in steel," ISIJ Int. 32, 350-358 (1992).

7. M. J. Luton, R. Dorvel, and R. A. Petkovic, "Interaction between deformation, recrystallization and precipitation in niobium steels," Metall. Trans. A 11, 411-420 (1980).

8. M. Gómez, L. Rancel, and S. F. Medina, "Effects of aluminium and nitrogen on static recrystallisation in V-microalloyed steels," Mater. Sci. Eng., A 506, 165173 (2009).

9. O. Kwon and A. DeArdo, "Interactions between recrystallization and precipitation in hot-deformed microalloyed steels," Acta Metall. Mater. 39, 529-538 (1990).

10. B. Dutta, E. Valdes, and C. M. Sellars, "Mechanisms and kinetics of strain induced precipitation of $\mathrm{Nb}(\mathrm{C}, \mathrm{N})$ in austenite," Acta Metall. Mater. 40, 653-662 (1992).

11. S. Verninckt, K. Verbeken, P. Thibaux, and Y. Houbaert, "Recrystallization- precipitation interaction during austenite hot deformation of a $\mathrm{Nb}$ microalloyed steel," Mater. Sci. Eng., A 528, 5519-5528 (2011).

12. I. Andersen and O. Grong, "Analytical modelling of grain-growth in metals and alloys in the presence of growing and dissolving precipitates .1. Normal graingrowth, "Acta Metall. Mater. 43, 2673-2688 (1995).

13. H. S. Zurob, Y. Brechet, and G. Purdy, "A model for the competition of precipitation and recrystallization in deformed austenite,"Acta Mater. 49, 4183-4190 (2001).

14. P. Maugis and M. Gouné, "Kinetics of vanadium carbonitride precipitation in steel: A computer model," Acta Mater. 53, 3359-3367 (2005).

15. M. Mukherjee, U. Prahl, and W. Bleck, "Modelling the strain-induced precipitation kinetics of vanadium carbonitride during hot working of precipitation-hardened of ferritic-pearlitic steels," Acta Mater. 71, 234-254 (2014).

16. B. Dutta, E. J. Palmiere, and C. M. Sellars, "Modelling the kinetics of strain induced precipitation in $\mathrm{Nb}$ microalloyed steels," Acta Mater. 49, 785-794 (2001).

17. K. C. Russel, "Nucleation in solids: the induction and steady state effects," Adv. Colloid Interface Sci. 13, 205-318 (1980).

18. R. Wagner, R. Kampmann, and P. W. Voorhees, "Homogeneous Second Phase Precipitation," in Phase Transformations in Materials, Materials Science Monographs, Ed. by G. Kostorz (Wiley-VCH, Weinheim, 1991).

19. S. F. Medina, A. Quispe, and M. Gomez, "New model for strain induced precipitation kinetics in microalloyed steels," Metall. Mater. Trans. A 45, 1524-1539 (2014).

20. M. Perez, M. Dumont, and D. Acevedo-Reyes, "Implementation of classical nucleation and growth theories for precipitation," Acta Mater. 56, 2119-2132 (2008).

21. N. Fujita and H. K. D. H. Bhadeshia, "Modelling precipitation of niobium carbide in austenite: multicomponent diffusion, capillarity and coarsening," Mater. Sci. Tecnol. 17, 403-408 (2001).
22. A. E. Salas-Reyes, I. Mejía, A. Bedolla-Jacuinde, A. Boulaajaj, J. Calvo, and J. M. Cabrera, "Hot ductility behavior of high-Mn austenitic $\mathrm{Fe}-22 \mathrm{Mn}-1.5 \mathrm{Al}-$ 1.5Si-0.45C TWIP steels microalloyed with Ti and V," Mater. Sci. Eng., A 611, 77-89 (2014).

23. I. Mejía, A. E. Salas-Reyes, A. Bedolla-Jacuinde, J. Calvo, and J. M. Cabrera, "Effect of $\mathrm{Nb}$ and Mo on the hot ductility behavior of a high-manganese austenitic Fe-21 Mn-1.3Al-1.5Si-0.5C TWIP steel," Mater. Sci. Eng., A 616, 229-239 (2014).

24. F. Perrard, A. Deschamps, and P. Maugis, "Modelling the precipitation of $\mathrm{NbC}$ on dislocations in $\alpha$-Fe," Acta Mater. 55, 1255-1266 (2007).

25. S. F. Medina, C. A. Hernández, and J. Ruiz, "Modelling austenite flow curves in low alloy and microalloyed steels," Acta Mater. 44, 155-163 (1996).

26. T. Gladman, The Physical Metallurgy of Microalloyed Steels (The Institute of Materials, London, 1997).

27. M. Perez, E. Courtois, D. Acevedo, T. Epicier, and P. Maugis, "Precipitation of niobium carbonitrides in ferrite: chemical composition measurements and thermodynamic modelling," Philos. Mag. Lett. 87, 645656 (2007).

28. M. Perez and A. Deschamps, "Microscopic modelling of simultaneous two phase precipitation: application to carbide precipitation in low carbon steels," Mater. Sci. Eng., A 360, 214-219 (2003).

29. H. Oikawa, "Lattice diffusion in iron-A review," Trans. Iron Steel Inst. Jpn. 68, 1489-1497 (1982).

30. J. Ardell, "The effect of volume fraction on particle coarsening: theoretical considerations," Acta Metall. 20, 61-71 (1972).

31. I. I. Gorbachev, V. V. Popov, and A. Yu. Pasynkov, "Calculations of the influence of alloying elements (Al, Cr, Mn, Ni, Si) on the solubility of carbonitrides in low-carbon low-alloy steels," Phys. Met. Metallogr. 117, 1277-1287 (2016).

32. A. Deschamps, and Y. Brechet, "Influence of predeformation and ageing of an $\mathrm{Al}-\mathrm{Zn}-\mathrm{Mg}$ alloy-II. Modelling of precipitation kinetics and yield stress," Acta Mater. 47, 293-305 (1999).

33. Z. Baochun, Z. Tan, L. Guiyan, and L. Qiang, "Metadynamic recrystallizaton behavior of a vanadium-nitrogen microalloyed steel," Met. Mater. Int. 21, 692697 (2015).

34. S. F. Medina, L. Rancel, M. Gómez, R. Ishak, and M. De Sanctis, "Intragranular nucleation of ferrite on precipitates and grain refinement in a hot deformed V-microalloyed steel," ISIJ Int. 48, 1063-1608 (2008).

35. M. Gomez, L. Rancel, and S. F. Medina, "Effects of aluminium and nitrogen on static recrystallisation in V-microalloyed steels," Mater. Sci. Eng., A, 506, 165173 (2009).

36. S. F. Medina, "From heterogeneous to homogeneous nucleation for precipitation in austenite of microalloyed steels," Acta Mater. 84, 202-207 (2015).

37. W. Hui, S. Chen, Y. Zhang, C. Shao, and H. Dong, "Effect of vanadium on the high-cycle fatigue fracture properties of medium-carbon microalloyed steel for fracture splitting connecting rod," Mater. Des. 66, 227-234 (2015).

38. V. V. Popov, I. I. Gorbachev, and J. A. Alyabieva, "Simulation of VC precipitate evolution in steels with consideration for the formation of new nuclei," Philos. Mag. 85, 2449-2467 (2005). 